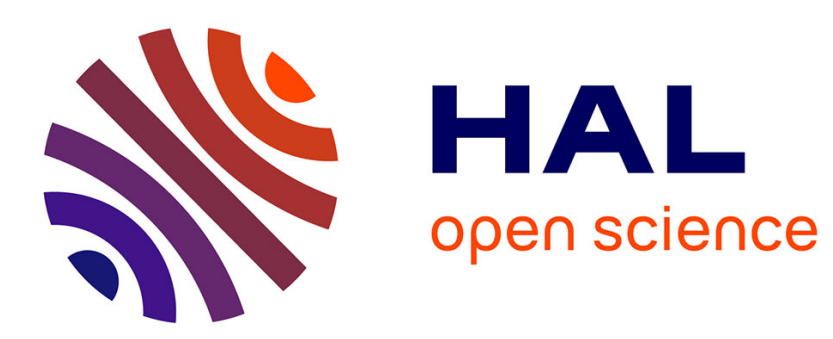

\title{
Reducing the number of neighbors in the received constellation of dmin precoded MIMO systems
}

Quoc-Tuong Ngo, Olivier Berder, Pascal Scalart

\section{To cite this version:}

Quoc-Tuong Ngo, Olivier Berder, Pascal Scalart. Reducing the number of neighbors in the received constellation of dmin precoded MIMO systems. Proc. of the IEEE Conference on Wireless Communications and Networking Conference (WCNC), Mar 2011, Cancun, Mexico, France. pp.1635 -1639. hal-00746409

\section{HAL Id: hal-00746409 \\ https://hal.science/hal-00746409}

Submitted on 29 Oct 2012

HAL is a multi-disciplinary open access archive for the deposit and dissemination of scientific research documents, whether they are published or not. The documents may come from teaching and research institutions in France or abroad, or from public or private research centers.
L'archive ouverte pluridisciplinaire HAL, est destinée au dépôt et à la diffusion de documents scientifiques de niveau recherche, publiés ou non, émanant des établissements d'enseignement et de recherche français ou étrangers, des laboratoires publics ou privés. 


\title{
Reducing the number of neighbors in the received constellation of $d_{\text {min }}$ precoded MIMO systems
}

\author{
Quoc-Tuong Ngo, Olivier Berder and Pascal Scalart \\ IRISA - Université de Rennes 1, France \\ Email: Firstname.Lastname@irisa.fr
}

\begin{abstract}
Not only the minimum Euclidean distance but also the number of neighbors providing the $d_{\min }$ in the received constellation has an important role in reducing the error probability when Maximum Likelihood detection is considered. Assuming that the channel-state-information is available at the transmitter, a new precoder in which the rotation parameter has no influence is proposed for two independent data-streams. The expression of the new precoding strategy is less complex and the space of solution is, therefore, smaller. Simulation results over Rayleigh fading channel confirm a bit-error-rate improvement of the proposed solution in comparison with other precoders. The improvement depends on the channel characteristics and is more significant if the channel is dispersive.
\end{abstract}

\section{INTRODUCTION}

In recent years, many communication techniques have been developed to adapt the demand for high data rates in wireless transmission. One of the most popular technique, known as multiple-input multiple-output (MIMO), provides a large gain in capacity and achieves a higher spectral efficiency compared to single antenna links [1]. Spatial multiplexing is a simple MIMO technique over which multiple independent datastreams are transmitted. At the receiver of spatial multiplexing system, a low error rate Maximum Likelihood (ML) detection is usually utilized. However its performance depends on the behavior of the matrix channel. If the channel state information (CSI) is available at the transmitter, the linear precoding technique, where the transmitted symbols are premultiplied by a precoding matrix, is proposed to significantly improve the performance of the ML detection [2].

Linear precoders can be used for a transmitter with full CSI [3], or limited feedback CSI [4] from the receiver. They are designed to optimize various criteria such as maximizing the minimum singular value [3], maximizing the post-processing SNR [5], and minimizing the mean square error (MSE) [6]. These precoding matrices are all diagonal and lead to power allocation strategies. The authors in [7], [8], [9] proposed a non-diagonal precoder which is based on the maximization of the minimum Euclidean distance $\left(d_{\min }\right)$ between two received symbols. The max- $d_{\min }$ precoder achieves a significant biterror-rate (BER) improvement in comparisons with diagonal precoders, especially when a ML detection is considered at the receiver.

In this paper, we propose a new version of maximum $d_{\min }$ based precoding, the $\mathrm{N}-d_{\text {min }}$ precoder. This precoding strategy considers not only the minimum Euclidean distance but also the number of neighbors providing it. Simulation results for
BPSK and QPSK modulations confirm the improvement of the new precoder on the BER performance in comparison with different traditional precoders.

The paper is organized as follows. Section II introduces the MIMO channel representation when a precoder is considered at the transmitter. The impact of the minimum Euclidean distance in the received constellation on the BER performance of the system is described in Section III. In Section IV, the optimization of the $d_{\text {min }}$ criterion which reduces the number of neighbors is detailed. Finally, we present in Section V the BER simulation results over a Rayleigh fading channel and the conclusions are given in Section VI.

\section{SYSTEM MODEL}

We consider a MIMO system with $n_{T}$ transmit and $n_{R}$ receive antennas. The system concerns the transmission of $b$ independent datastreams over Rayleigh fading channel. The received signal is then given by

$$
\mathbf{y}=\mathbf{G H F s}+\mathbf{G} \nu
$$

where $\mathbf{H}$ is the $n_{R} \times n_{T}$ channel matrix, $\mathbf{F}$ is the $n_{T} \times b$ precoding matrix, $\mathbf{G}$ is the $b \times n_{R}$ decoding matrix, $\mathbf{s}$ is the $b \times 1$ transmitted vector symbol, and $\nu$ is the $n_{R} \times 1$ additive Gaussian noise vector.

If perfect channel state information (CSI) is considered at both the transmitter and receiver, it was shown that the channel matrix can be diagonalized by using a virtual transformation [7]. The precoder and decoder matrices are then decomposed as $\mathbf{F}=\mathbf{F}_{v} \mathbf{F}_{d}$ and $\mathbf{G}=\mathbf{G}_{d} \mathbf{G}_{v}$. The received signal now can be re-expressed as

$$
\mathbf{y}=\mathbf{G}_{d} \mathbf{H}_{v} \mathbf{F}_{d} \mathbf{s}+\mathbf{G}_{d} \nu_{v}
$$

where $\mathbf{H}_{v}=\mathbf{G}_{v} \mathbf{H F}_{v}=\operatorname{diag}\left(\sigma_{1}, \ldots, \sigma_{b}\right)$ is the $b \times b$ virtual channel matrix, $\nu_{v}=\mathbf{G}_{v} \nu$ is the $b \times 1$ transformed additive Gaussian noise vector.

In the paper, an ML detection is considered at the receiver so the decoder matrix $\mathbf{G}_{d}$ has no effect on the performance and is consequently assumed to be an identity matrix of size $b$. Therefore, the received signal can be simplified as

$$
\mathbf{y}=\mathbf{H}_{v} \mathbf{F}_{d} \mathbf{s}+\nu_{v} .
$$

Let us note $E_{s}$ as the average transmit power. The power constraint is

$$
\operatorname{trace}\left\{\mathbf{F}_{d} \mathbf{F}_{d}^{*}\right\}=E_{s}
$$


The precoding matrix $\mathbf{F}_{d}$ is designed to adapt various forms of channel information. In the next section, we consider the impact of the minimum Euclidean distance on the error probability of the precoded MIMO system.

\section{Minimum Euclidean Distance ON PRECOdeR}

Let us define a vector $\mathbf{x}=\mathbf{H}_{v} \mathbf{F}_{d} \mathbf{s}$, and denote by $A_{i j}$ the event that $\left\|\mathbf{y}-\mathbf{x}_{j}\right\|<\left\|\mathbf{y}-\mathbf{x}_{i}\right\|$ when the symbol $\mathbf{s}_{i}$ was sent at the transmitter. If the event $A_{i j}$ happens, there is an error detection. The received constellation is decoded correctly if $\left\|\mathbf{y}-\mathbf{x}_{i}\right\|<\left\|\mathbf{y}-\mathbf{x}_{j}\right\|$ with $\forall j \neq i$ when $\mathbf{s}_{i}$ was sent. Then the average error probability can be defined by

$$
P_{e}=\frac{1}{M} \sum_{i=1}^{M} P_{e_{i}}\left\{\mathbf{s}_{i} \text { sent }\right\}=\frac{1}{M} \sum_{i=1}^{M} \operatorname{Prob}\left\{\bigcup_{\substack{j=1 \\ j \neq i}}^{M} A_{i j}\right\}
$$

where $M$ is the number of all possible transmitted vectors $\mathbf{s}$. The average error probability can be approximated by

$$
P_{e} \simeq \frac{1}{M} \sum_{i=1}^{M} \sum_{\substack{j=1 \\ j \neq i}}^{M} \operatorname{Prob}\left\{A_{i j}\right\}
$$

where

$$
\begin{aligned}
\operatorname{Prob}\left\{A_{i j}\right\} & =\operatorname{Prob}\left\{\left\|\mathbf{y}-\mathbf{x}_{j}\right\|<\left\|\mathbf{y}-\mathbf{x}_{i}\right\| \mid \mathbf{s}_{i} \text { sent }\right\} \\
& =\operatorname{Prob}\left\{\left\|\mathbf{x}_{i}+\nu_{v}-\mathbf{x}_{j}\right\|<\left\|\mathbf{x}_{i}+\nu_{v}-\mathbf{x}_{i}\right\|\right\} \\
& =\operatorname{Prob}\left\{\left\|\nu_{v}-\left(\mathbf{x}_{j}-\mathbf{x}_{i}\right)\right\|<\left\|\nu_{v}\right\|\right\} .
\end{aligned}
$$

Let us define $d_{i j}=\left\|\mathbf{x}_{j}-\mathbf{x}_{i}\right\|$ and $n_{v}$ the projection of vector $\nu_{v}$ onto the vector $\left(\mathbf{x}_{j}-\mathbf{x}_{i}\right)$, we have

$$
\begin{aligned}
& \operatorname{Prob}\left\{\left\|\nu_{v}-\left(\mathbf{x}_{j}-\mathbf{x}_{i}\right)\right\|<\left\|\nu_{v}\right\|\right\}=\operatorname{Prob}\left\{n_{v}>\frac{d_{i j}}{2}\right\} \\
= & Q\left(\frac{d_{i j}}{2 \sqrt{N_{0}}}\right)=Q\left(\frac{\bar{d}_{i j}}{2 \sqrt{N_{0}}} \times \sqrt{E_{s}}\right)
\end{aligned}
$$

where $N_{0}$ is the variance of the white Gaussian noise $\nu_{v}$, and $\bar{d}_{i j}$ is the normalized distance of vector $\left(\mathbf{x}_{j}-\mathbf{x}_{i}\right)$.

Therefore, the error probability can be simplified as

$$
P_{e} \simeq \frac{1}{M} \sum_{i=1}^{M} \sum_{\substack{j=1 \\ j \neq i}}^{M} Q\left(\frac{\bar{d}_{i j}}{2 \sqrt{N_{0}}} \times \sqrt{E_{s}}\right) .
$$

According to (7), we can appreciate the impact of the Euclidean distances on the BER performance of a MIMO system. Let us first consider the simplest case: there are only two Euclidean distances.

Lemma 1: For every $d_{\alpha}<d_{\beta}<d_{\chi}<d_{\delta}$, we can find the value of $\mathrm{R}$ high enough satisfying the condition

$$
Q\left(d_{\alpha} \cdot R\right)+Q\left(d_{\delta} \cdot R\right)>Q\left(d_{\beta} \cdot R\right)+Q\left(d_{\chi} \cdot R\right)
$$

Proof: see Appendix A.

It is obvious that we can improve the BER performance by increasing the minimum Euclidean distance of the received constellation. One should note that $Q\left(d_{\beta} \cdot R\right)>Q\left(d_{\chi} \cdot R\right)$, so $\forall d_{\chi}$ such that $d_{\alpha}<d_{\chi}<d_{\delta}$, we can obtain

$$
Q\left(d_{\alpha} \cdot R\right)+Q\left(d_{\delta} \cdot R\right)>2 Q\left(d_{\chi} \cdot R\right) .
$$

This is an actual evidence that the optimized detection, in reality, is obtained when the minimum distance is reached by many Euclidean distances.

Lemma 2: With two arrays $d_{\alpha_{i}}$ and $d_{\beta_{i}}$ which are sorted by increasing order, if $d_{\alpha_{1}}<d_{\beta_{1}}$ and $k \geq 2$, we can find the value of $\mathrm{R}$ high enough such that

$$
\sum_{i=1}^{k} Q\left(d_{\alpha_{i}} \cdot R\right)>\sum_{i=1}^{k} Q\left(d_{\beta_{i}} \cdot R\right)
$$

Proof: see Appendix B.

From the form of error probability in (7) and the remark in the Lemma 2, it can be concluded that the minimum Euclidean distance has a very important role in the BER improvement of the precoding strategies system. We can predict that the optimized precoder can be obtained when the minimum Euclidean distance on the received constellation is provided by many difference vectors.

\section{IV. $\mathrm{N}-d_{\text {min }}$ PRECODER}

Let us note $\mathrm{N}_{i}$ is the number of distances $\bar{d}_{i j}$ such that $\bar{d}_{i j}=d_{\min }$ where the minimum Euclidean distance $d_{\min }$ is defined by

$$
d_{\text {min }}^{2}=\min _{s_{k}, s_{l} \in S, s_{k} \neq s_{l}}\left\|\mathbf{H}_{v} \mathbf{F}_{d}\left(\mathbf{s}_{k}-\mathbf{s}_{l}\right)\right\|^{2} .
$$

A numerical search over $\mathbf{F}_{d}$, which maximizes the minimum Euclidean distance obtained by many difference vectors, shows that the values of other Euclidean distances are much higher than the minimum distance when $d_{\min }$ is optimized. In that case, the other distances have no much impact on the biterror-rate performance. The error probability in (7) can be then simplified as

$$
\begin{aligned}
P_{e} & \approx \frac{1}{M} \sum_{i=1}^{M} \mathrm{~N}_{i} \cdot Q\left(\frac{\bar{d}_{\text {min }}}{2 \sqrt{N_{0}}} \times \sqrt{E_{s}}\right) \\
& \approx \mathrm{N}_{d_{\min }} \cdot Q\left(\frac{\bar{d}_{\min }}{2 \sqrt{N_{0}}} \times \sqrt{E_{s}}\right)
\end{aligned}
$$

where $\mathrm{N}_{d_{\min }}=\frac{1}{M} \sum_{i=1}^{M} \mathrm{~N}_{i}$. It is observed that to improve the BER performance of the precoding strategies system, we have to not only maximize the minimum Euclidean distance but also minimize the number of neighbors providing it. The new precoding strategy is, therefore, called as $\mathrm{N}-d_{\min }$ precoder.

To illustrate the method of optimization, let us consider a simple case: $b=2$. By using a singular value decomposition, the authors in [7] and [8] simplified the virtual channel and precoding matrices as

$$
\mathbf{H}_{v}=\left(\begin{array}{cc}
\sigma_{1} & 0 \\
0 & \sigma_{2}
\end{array}\right)=\rho\left(\begin{array}{cc}
\cos \gamma & 0 \\
0 & \sin \gamma
\end{array}\right)
$$

where $\rho=\sqrt{\sigma_{1}^{2}+\sigma_{2}^{2}}$ and $\gamma=\arctan \frac{\sigma_{2}}{\sigma_{1}}$ are the channel gain and channel angle, respectively.

$$
\mathbf{F}_{d}=\sqrt{E_{s}}\left(\begin{array}{cc}
\cos \psi & 0 \\
0 & \sin \psi
\end{array}\right)\left(\begin{array}{cc}
\cos \theta & \sin \theta \\
-\sin \theta & \cos \theta
\end{array}\right)\left(\begin{array}{ll}
1 & 0 \\
0 & e^{i \varphi}
\end{array}\right)
$$

with $0 \leq \theta \leq \pi / 4$ and $0 \leq \psi, \varphi \leq \pi / 2$. The parameter $\psi$ controls the power allocation on the virtual subchannels, 
$\theta$ and $\varphi$ correspond to scaling and rotation of the received constellation, respectively. When $\theta$ and $\varphi$ are equal to 0 , the precoding matrix is diagonal and equivalent to the power allocation strategies.

We present, herein, an original idea not only considering $d_{\min }$ and $\mathrm{N}_{d_{\min }}$, simultaneously, but also reducing the complexity of the solution. It is realized that if the coefficients of the precoding matrix $\mathbf{F}_{D}$ do not depend on the rotation parameter $(\varphi=0$ or $\varphi=\pi / 2)$, the received constellation will have less distances which can reach the minimum Euclidean distance. The property could be explained by the non-rotated received constellation (or perpendicular rotated constellation) when a Quadrature Amplitude Modulation (QAM) is used at the transmitter.

For this reason, we propose a new precoding strategy in which we assume that the rotation parameter has no employ ( $\varphi=0$ or $\varphi=\pi / 2)$. By using the parameterized form of the precoder in (14), we are now looking for the angles $\psi$ and $\theta$ which optimize the $d_{\text {min }}$ criterion for each channel angle $\gamma$. A numerical approach for MIMO system using BPSK and QPSK modulation, which is considered in the following of this section, confirms a bit-error-rate improvement of our new precoder.

\section{A. For BPSK modulation}

It is observed that the difference vector as given by the difference between the two transmitted vectors $\left(\breve{\mathbf{s}}=\mathbf{s}_{k}-\mathbf{s}_{l}\right.$ with $\mathbf{s}_{k} \neq \mathbf{s}_{l}$ ) is a vector created by the elements of the set $\{0,2,-2\}$. A numerical search over $\psi$ and $\theta$ which optimize the minimum Euclidean distance for two independent datastreams shows that the $\mathrm{N}-d_{\min }$ precoder has the same form as the max- $d_{\text {min }}$ precoder presented in [7]

$$
\mathbf{F}_{D}=\sqrt{\frac{E_{s}}{2}}\left(\begin{array}{ll}
1 & i \\
0 & 0
\end{array}\right) \text {. }
$$

One should note that the $\mathrm{N}-d_{\min }$ solution pours power only on the strongest virtual sub-channels. The minimum Euclidean distance is then defined by

$$
d_{B P S K}^{2}=4 E_{s} \rho^{2} \cos ^{2} \gamma .
$$

\section{B. For QPSK modulation}

The transmitted symbols belong to the following set $S=$ $\frac{1}{\sqrt{2}}\{1+i, 1-i,-1+i,-1-i\}$. For QPSK modulation with two datastreams, the set of all difference vectors denoted as $\breve{\mathbf{S}}_{Q P S K}$ contains $16 \times 15=240$ elements. By eliminating the collinear vectors, we can reduce the size of $\breve{\mathbf{S}}_{Q P S K}$ to 14 elements.

A numerical search over $\psi$ and $\theta$ which optimize the minimum Euclidean distance for each channel angle, shows that our precoder has two different expressions. The first one, denoted as $\mathbf{F}_{s n r}$ pours power only on the strongest virtual subchannel. The other, obviously, uses all two virtual subchannels to transmit symbols, and is denoted as $\mathbf{F}_{r e c}$.
1) For the first expression: the power is concentrated only on the first virtual subchannel and the rotation parameter $\varphi$ is not considered at the precoder. The form of the precoder $\mathbf{F}_{s n r}$ can be expressed as

$$
\mathbf{F}_{s n r}=\sqrt{\frac{E_{s}}{5}}\left(\begin{array}{ll}
2 & 1 \\
0 & 0
\end{array}\right) .
$$

The optimized $d_{\min }$ is provided by the difference vector $\frac{1}{\sqrt{2}}[02]^{T}$, and defined by

$$
d_{s n r}^{2}=\frac{2}{5} E_{s} \rho^{2} \cos ^{2} \gamma .
$$

The received constellation obtained by $\mathbf{F}_{s n r}$ looks like the 16-QAM constellation. Hence, the average number of neighbors providing $d_{\min }$ is given by $\mathrm{N}_{d_{\min }}=\frac{1}{16}(4 \times 2+$ $8 \times 3+4 \times 4)=3$. This value is less than the number of the minimum Euclidean distances obtained by the precoder $\mathbf{F}_{r 1}$ presented in [7] $\left(\mathrm{N}_{d_{\min }}=\frac{1}{16}(4 \times 2+4 \times 3+4 \times 4+4 \times 5)=3.5\right)$. However, the distances $d_{\text {min }}$ provided by two precoders remain very close. This explains why the new precoder has a slight improvement of BER in comparison with the max- $d_{\min }$ precoder (see section $\mathrm{V}$ ).

2) For the second expression: the difference between two virtual subchannels is smaller than the case of $\mathbf{F}_{\text {snr }}$. A numerical search shows that the optimized solution is found when the angle $\theta=\pi / 4$ is fixed and $\psi$ depends on the channel angle $\gamma$. Indeed, the optimization is obtained by three difference vectors

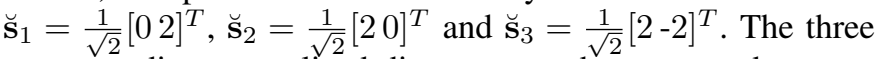
corresponding normalized distances can be expressed as

$$
\left\{\begin{array}{l}
\bar{d}_{\breve{x}_{1}}^{2}=A \sin ^{2} \theta+B \cos ^{2} \theta \\
\bar{d}_{\widetilde{x}_{2}}^{2}=A \cos ^{2} \theta+B \sin ^{2} \theta \\
\bar{d}_{\breve{x}_{3}}^{2}=A(\cos \theta-\sin \theta)^{2}+B(\cos \theta+\sin \theta)^{2}
\end{array}\right.
$$

where $A=4 \cos ^{2} \gamma \cos ^{2} \psi$ and $B=4 \sin ^{2} \gamma \sin ^{2} \psi$. By considering $\bar{d}_{\widetilde{x}_{1}}^{2}=\bar{d}_{\breve{x}_{2}}^{2}=\bar{d}_{\widetilde{x}_{3}}^{2}$ in the interval value of $\theta$ and $\psi$, we obtain

$$
\left\{\begin{array}{l}
\theta=\pi / 4 \\
\psi=\operatorname{atan} \frac{1}{\sqrt{3} \cdot \tan \gamma}
\end{array}\right.
$$
by

By substituting (19) into (14), the precoder $\mathbf{F}_{r e c}$ is given

$$
\mathbf{F}_{r e c}=\sqrt{\frac{E_{s}}{2}}\left(\begin{array}{cc}
\cos \psi & 0 \\
0 & \sin \psi
\end{array}\right)\left(\begin{array}{rr}
1 & 1 \\
-1 & 1
\end{array}\right) .
$$

The minimum Euclidean distance provided by $\mathbf{F}_{r e c}$ is then

$$
d_{r e c}^{2}=E_{s} \rho^{2} \frac{4 \sin ^{2} \gamma}{3 \tan ^{2} \gamma+1}
$$

Fig. 1 illustrates the received constellation of the precoder for QPSK modulation. It is observed that the average number of $d_{\min }$ is defined by $\mathrm{N}_{d_{\min }}=\frac{1}{16}(4 \times 4+8 \times 5+4 \times 6)=5$. In comparison with the precoder in [7] where $\mathrm{N}_{d_{\min }}=\frac{1}{16}(8 \times$ $5+8 \times 9)=7$, our new precoder has a good improvement. 


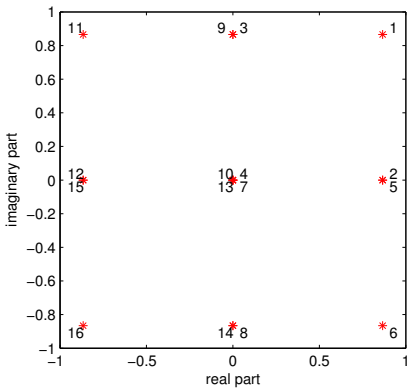

(a) First virtual subchannel

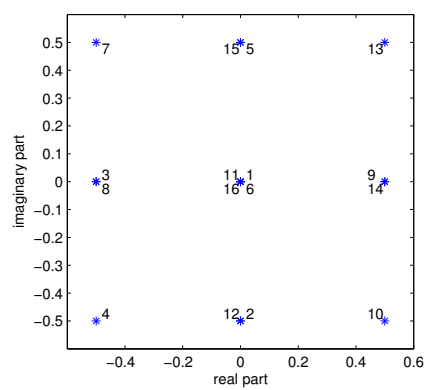

(b) Second virtual subchannel
Fig. 1: Received constellation of the precoder $\mathbf{F}_{r e c}$ for QPSK.

3) The threshold $\gamma_{0}$ : To choose between $\mathbf{F}_{s n r}$ and $\mathbf{F}_{r e c}$, we have to compare the error probabilities in (7) which are obtained by both precoders. It is observed that when the channel angle $\gamma$ varies from 0 to $\pi / 2$, the ratios of other distances to the distance $d_{\min }$ are fixed. Furthermore, we realize that the minimum Euclidean distance $d_{s n r}$ and $d_{r e c}$ in $(18,21)$ is proportional to $E_{s}$. For this reason, the threshold $\gamma_{0}$ is not constant and depends on the signal-to-noise ratio $\sqrt{E_{s} / N_{0}}$. The angle $\gamma_{0}$ increases to $\gamma_{c}$ if the average transmit power $E_{s}$ augments. The critical angle $\gamma_{c}$ is given by

$$
\begin{aligned}
& d_{s n r}^{2}=d_{r e c}^{2} \\
\Leftrightarrow & \frac{2}{5} \cos ^{2} \gamma_{c}=4 \frac{\sin ^{2} \gamma_{c}}{3 \tan ^{2} \gamma_{c}+1} \\
\Leftrightarrow & \gamma_{c}=\operatorname{atan} \sqrt{1 / 7} .
\end{aligned}
$$

\section{Simulation RESUlts}

Fig. 2 illustrates the normalized minimum Euclidean distance $d_{\min }$ of the new precoder $\mathrm{N}-d_{\min }$ and other precoders in the case of QPSK modulation. The average transmit power $E_{s}$ for diagonal precoders is choosen large enough such that the power is always allocated on both virtual subchannels. It is observed that the $\mathrm{N}-d_{\min }$ solution is better than WaterFiling [1], $\max -\lambda_{\min }$ [3] and MMSE [6] precoders in terms of $d_{\min }$. The new precoder has a small difference of $d_{\min }$ in comparison with $\max -d_{\min }$ precoder [7]. Furthermore, the difference remains constant for a small channel angle $\left(\gamma<17.28^{\circ}\right)$.

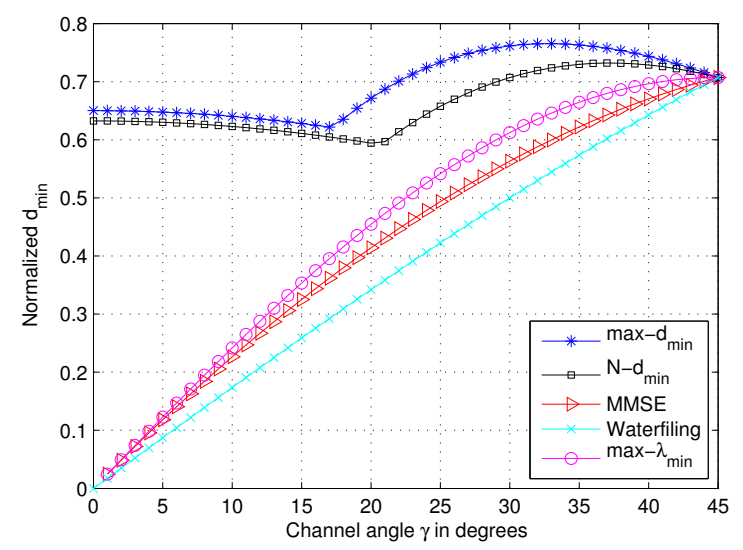

Fig. 2: Normalized minimum Euclidean distance for QPSK.
According to the improvement of not only the minimum Euclidean distance (except for max- $d_{\min }$, of course) but also the average number of $d_{\mathrm{min}}$, an increase of BER performance for $\mathrm{N}-d_{\min }$ precoder is expected for QPSK modulation. We consider a MIMO-OFDM system with $n_{T}=3$ transmit antennas and $n_{R}=2$ receive antennas, 128 subcarriers which occupy a bandwidth of $19 \mathrm{MHz}$. The channel matrix $\mathbf{H}$ is complex Gaussian and the noise element are additive white Gaussian.

Firstly, we compare the BER performances obtained by the new precoder $\mathrm{N}-d_{\min }$ and the $\max -d_{\min }$ solution. Fig. 3 shows a BER improvement of the precoder $\mathbf{F}_{s n r}$ and $\mathbf{F}_{r e c}$ in comparison with $\mathbf{F}_{r 1}$ and $\mathbf{F}_{\text {octa }}$ for small and large channel angle $\gamma$, respectively. We observe a large BER improvement of the precoder $\mathbf{F}_{r e c}$ compared to $\mathbf{F}_{\text {octa }}$, and a slight superiority of $\mathbf{F}_{s n r}$ in comparison with $\mathbf{F}_{r 1}$, although both new precoders are inferior in terms of $d_{\min }$. This result clearly demonstrates that the number of minimum Euclidean distances has an important role in reducing the error probability when an ML detection is considered at the receiver.

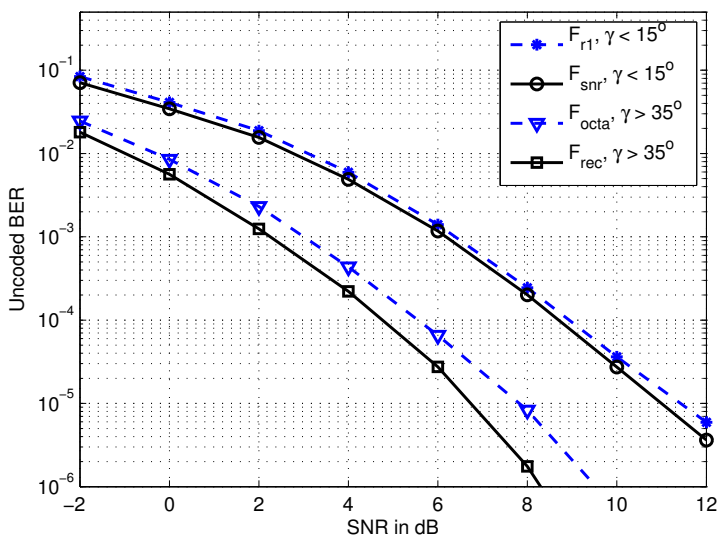

Fig. 3: BER comparison of $\max -d_{\min }$ and $\mathrm{N}-d_{\min }$ precoders.

The BER performance with respect to SNR for QPSK modulation is plotted in Fig. 4. One should note that the expression of $\mathrm{N}-d_{\min }$ precoder is associated to the max- $d_{\min }$ solution with $\mathbf{F}_{s n r}$ and $\mathbf{F}_{r e c}$ are available in large and small dispersion of virtual channel, respectively. As expected, the $\mathrm{N}-d_{\text {min }}$ precoder provides a significant improvement in term of BER compared to diagonal precoders. Furthermore, it has a slight improvement in comparison with $\max -d_{\min }$ precoder. This can be explained by the distribution of the channel angles $\gamma$ : the MIMO system $(3,2)$ uses more often the precoder $\mathbf{F}_{\text {snr }}$ than $\mathbf{F}_{r e c}$.

\section{CONCLUSION}

In the first part of this paper, we investigated the impact of the minimum Euclidean distance on the performance of biterror-rate when a ML detection is considered at receive side. It is realized that the number neighbors providing $d_{\min }$ has an important role in reducing the error probability. Therefore, a new precoder for MIMO transmission, which is based on the 


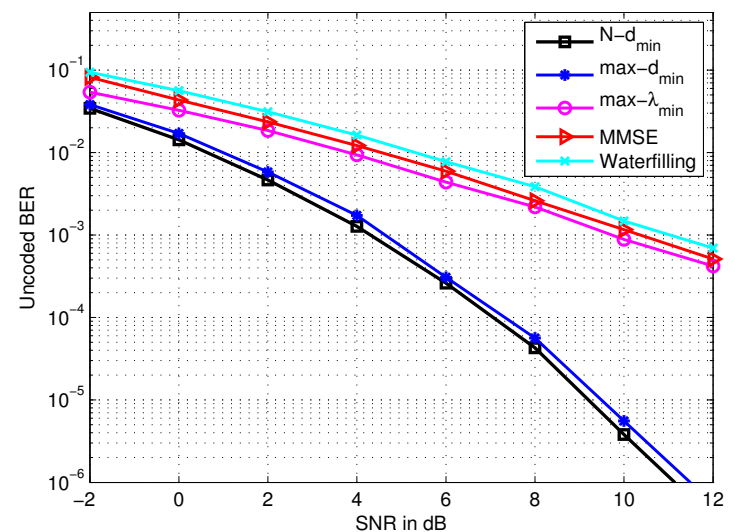

Fig. 4: Uncoded BER performance for QPSK modulation.

maximization of $d_{\min }$ associated with the minimization of the neighbors providing it, has been introduced.

In the new precoding strategy, the rotation parameter $\varphi$ is not considered. Hence, the degree of freedom in precoding matrix $\mathbf{F}_{d}$ is decreased and the space of the solution is smaller. Not only reducing the complexity, the $\mathrm{N}-d_{\text {min }}$ precoder presents also a significant improvement of BER compared to diagonal precoders such as MMSE, Waterfiling and $\max -\lambda_{\min }$. In comparison with $\max -d_{\min }$ precoder, the new precoder provides a slight improvement. The BER enhancement depends on channel characteristics and is more significant if the virtual subchannels are far from dispersive.

\section{APPENDIX A}

\section{PROOF OF LEMMA 1}

For all $d_{\beta}<d_{\chi}$, it is obvious that we can find a high value of $R$ such that

$$
d_{\chi}^{2}-d_{\beta}^{2}+\frac{2}{R^{2}} \log \frac{d_{\beta}-d_{\alpha}}{d_{\delta}-d_{\chi}}>0
$$

with $d_{\beta}>d_{\alpha}$ and $d_{\delta}>d_{\chi}$. The inequality (23) can be rewritten as

$$
\begin{aligned}
& \log \frac{d_{\beta}-d_{\alpha}}{d_{\delta}-d_{\chi}}>-\left(d_{\chi}^{2}-d_{\beta}^{2}\right) \cdot R^{2} / 2 \\
\Leftrightarrow & \frac{d_{\beta}-d_{\alpha}}{d_{\delta}-d_{\chi}}>e^{-\left(d_{\chi}^{2}-d_{\beta}^{2}\right) \cdot R^{2} / 2} \\
\Leftrightarrow & R\left(d_{\beta}-d_{\alpha}\right) \cdot e^{-d_{\beta}^{2} \cdot R^{2} / 2}>R\left(d_{\delta}-d_{\chi}\right) \cdot e^{-d_{\chi}^{2} \cdot R^{2} / 2}
\end{aligned}
$$

Using the monotonic decreasing property of the function $e^{-x^{2} / 2}$, we obtain

$$
\begin{aligned}
& \int_{d_{\alpha} \cdot R}^{d_{\beta} \cdot R} e^{-x^{2} / 2} d x>\left(d_{\beta} \cdot R-d_{\alpha} \cdot R\right) \cdot e^{-\left(d_{\beta} \cdot R\right)^{2} / 2} \\
& \left(d_{\delta} \cdot R-d_{\chi} \cdot R\right) \cdot e^{-\left(d_{\chi} \cdot R\right)^{2} / 2}>\int_{d_{\chi} \cdot R}^{d_{\delta} \cdot R} e^{-x^{2} / 2} d x
\end{aligned}
$$

From (24), (25), and (26) we have

$$
\begin{gathered}
\int_{d_{\alpha} \cdot R}^{d_{\beta} \cdot R} e^{-x^{2} / 2} d x>\int_{d_{\chi} \cdot R}^{d_{\delta} \cdot R} e^{-x^{2} / 2} d x \\
\Leftrightarrow Q\left(d_{\alpha} \cdot R\right)-Q\left(d_{\beta} \cdot R\right)>Q\left(d_{\chi} \cdot R\right)-Q\left(d_{\delta} \cdot R\right) \\
\Leftrightarrow Q\left(d_{\alpha} \cdot R\right)+Q\left(d_{\delta} \cdot R\right)>Q\left(d_{\beta} \cdot R\right)+Q\left(d_{\chi} \cdot R\right) .
\end{gathered}
$$

\section{APPENDiX B}

PROOF OF LEMMA 2

The mathematical induction can be used to prove this lemma. One should note that $\mathrm{Q}(\mathrm{x})$ is a monotonic decreasing function. First we show that our statement holds for $k=2$. Indeed, there are two cases:

i) $d_{\alpha_{2}} \leq d_{\beta_{2}}$ : it is obvious that $Q\left(d_{\alpha_{1}} \cdot R\right)>Q\left(d_{\beta_{1}} \cdot R\right)$ and $Q\left(d_{\alpha_{2}} \cdot R\right) \geq Q\left(d_{\beta_{2}} \cdot R\right)$ with $\forall R>0$, so we have

$$
Q\left(d_{\alpha_{1}} \cdot R\right)+Q\left(d_{\alpha_{2}} \cdot R\right)>Q\left(d_{\beta_{1}} \cdot R\right)+Q\left(d_{\beta_{2}} \cdot R\right)
$$

ii) $d_{\alpha_{2}}>d_{\beta_{2}}$ : obviously, this is the case of the Lemma 1 .

Thus it has been shown that the lemma holds for $k=2$. We assume that our statement is true for $k$. It must then be shown that our statement is true for $k+1$. Let us define $d_{\gamma_{1}}=\frac{1}{3}\left(d_{\alpha_{1}}+\right.$ $\left.d_{\beta_{1}}\right)$, and $d_{\gamma_{2}}=\frac{2}{3}\left(d_{\alpha_{1}}+d_{\beta_{1}}\right)$. It is clear that $d_{\alpha_{1}}<d_{\gamma_{1}}<d_{\gamma_{2}}$, so we can find value $R_{1}$ such that $\forall R \geq R_{1}$

$$
Q\left(d_{\alpha_{1}} \cdot R\right)+Q\left(d_{\alpha_{2}} \cdot R\right)>Q\left(d_{\gamma_{1}} \cdot R\right)+Q\left(d_{\gamma_{2}} \cdot R\right)
$$

Since $d_{\gamma_{2}}<d_{\beta_{1}} \leq d_{\beta_{2}}$, so we get

$$
Q\left(d_{\gamma_{2}} \cdot R\right)>Q\left(d_{\beta_{2}} \cdot R\right) \text {. }
$$

Furthermore, we have $d_{\gamma_{1}}<d_{\beta_{1}}$. According to the statement in the case of $k$, there exist values $R_{2}$ which satisfies $\forall R \geq R_{2}$

$$
Q\left(d_{\gamma_{1}} \cdot R\right)+\sum_{i=3}^{k+1} Q\left(d_{\alpha_{i}} \cdot R\right)>Q\left(d_{\beta_{1}} \cdot R\right)+\sum_{i=3}^{k+1} Q\left(d_{\beta_{i}} \cdot R\right) .
$$

From (27), (28) and (29), it can be concluded that $\forall R \geq$ $\max \left(R_{1}, R_{2}\right)$, we have

$$
\sum_{i=1}^{k+1} Q\left(d_{\alpha_{i}} \cdot R\right)>\sum_{i=1}^{k+1} Q\left(d_{\beta_{i}} \cdot R\right) .
$$

\section{REFERENCES}

[1] E. Telatar, "Capacity of multi-antenna Gaussian channels," European transactions on telecommunications, vol. 10, no. 6, pp. 585-595, 1999.

[2] M. Vu and A. Paulraj, "MIMO wireless linear precoding," IEEE Signal Processing Magazine, vol. 24, no. 5, pp. 86-105, 2007.

[3] A. Scaglione, P. Stoica, S. Barbarossa, G. Giannakis, and H. Sampath, "Optimal designs for space-time linear precoders and decoders," IEEE Transactions on Signal Processing, vol. 50, no. 5, pp. 1051-1064, 2002.

[4] D. Love and R. Heath, "Limited feedback unitary precoding for spatial multiplexing systems," Information Theory, IEEE Transactions on, vol. 51, no. 8, pp. 2967-2976, 2005.

[5] P. Stoica and G. Ganesan, "Maximum-SNR spatial-temporal formatting designs for MIMO channels," IEEE Transactions on Signal Processing, vol. 50, no. 12, pp. 3036-3042, 2002.

[6] H. Sampath, P. Stoica, and A. Paulraj, "Generalized linear precoder and decoder design for MIMO channels using the weighted MMSE criterion," IEEE Transactions on Communications, vol. 49, no. 12, pp. 2198-2206, 2001.

[7] L. Collin, O. Berder, P. Rostaing, and G. Burel, "Optimal minimum distance-based precoder for MIMO spatial multiplexing systems," IEEE Transactions on Signal Processing, vol. 52, no. 3, pp. 617-627, 2004.

[8] Q.-T. Ngo, O. Berder, B. Vrigneau, and O. Sentieys, "Minimum distance based precoder for mimo-ofdm systems using 16-qam modulation," IEEE International Conference on Communications, ICC Dresden, Germany, pp. $1-5$, June 2009.

[9] Q.-T. Ngo, O. Berder, and P. Scalart, "3-d minimum euclidean distance based sub-optimal precoder for mimo spatial multiplexing systems," IEEE International Conference on Communications, ICC Cape Town, South Africa, May 2010. 